\title{
The use of specialised enteral formulae for patients with diabetes mellitus
}

\section{Abstract}

The majority of enteral nutrition products for diabetes mellitus have a carbohydrate content of $30-45 \%$ and fat between $40-49 \%$, mainly monounsaturated fat, with a mix of soluble and insoluble fibre (total of 14-24 g/l). Does this have short- and long-term benefits and which component(s) is/are crucial for the outcome or is it the combination that counts? Both manipulations of DM specific enteral formulae, i.e. addition of fibre and altered carbohydrate to fat percentage seem to be effective for short-term glucose control, but do not show convincing evidence regarding lipid management. In terms of gastro-intestinal function, there seems to be adequate evidence that fibre plays an important role for the management of diarrhoea and constipation. The implications of high fat intake on the longer-term, especially in patients suffering from gastroparesis, are less clear.

S Afr J Clin Nutr 2010;23(1) Supplement:S55-S57

\section{Introduction}

The medical nutrition therapy of a patient with diabetes mellitus (DM) poses many challenges to the health care provider. In order to reach the goals of treatment, careful consideration of the different treatment options needs to be investigated.

The goals of nutritional therapy for DM include the maintenance of as near-normal blood glucose levels as possible, the achievement of optimal blood lipid levels, the prevention and treatment of acute and chronic complications of diabetes; while concurrently maintaining fluid balance in a patient receiving nutritional support., ${ }^{1,2}$ Another important factor in the nutritional management of a diabetic patient is glycaemic control during enteral feeding since hyperglycaemia has been associated with an increased risk for infection in patients with DM and in ICU patients in general., ${ }^{2,3}$ Current guidelines for blood glucose control in critically ill patients with and without $\mathrm{DM}$, recommend keeping glucose values in the range of $6,1-8,3$ $\mathrm{mmol} / \mathrm{L} .{ }^{3,4}$ There is a higher incidence of severe hypoglycaemia in patients treated to tighter limits when blood glucose is maintained between 4.5 and $6.1 \mathrm{mmol} / \mathrm{L}^{4}$

\section{Diet composition}

The ideal composition of the diabetic diet, specifically with regards to the carbohydrate and fat content, has received much attention and research in the last 15 years. From a high carbohydrate, low fat diet to a low carbohydrate, high fat diet, to a mixture of both. What has become more evident with time is that it is not only the total amounts, but rather the type of carbohydrate and fat that is important. $^{1}$
The nutritional recommendations for individuals with DM of the American Diabetes Association for instance (Table I) indicate that in the 1990's, the recommended contribution to total energy (TE) of carbohydrate was $50-60 \%$, fat had to be $<30 \%$ and no recommendations were made for glycaemic index (Gl). ${ }^{5}$ About 10 12 years later, the recommendations changed to the combination of carbohydrate and fat, especially monounsaturated fat (MUFA), contributing between $60-70 \%$ total energy, saturated fat $<7 \%$, poly-unsaturated fat $<10 \%$, trans fatty acids as low as possible and cholesterol $<300 \mathrm{ml}$ per day. Still no Gl recommendation was included. ${ }^{6}$ From 2006 up to the present, no specific percentage recommendations are made for the percentage contribution of carbohydrate and fat in terms of total energy, but carbohydrate should be $>130 \mathrm{~g}$ per day, saturated fat $<7 \%$, trans fatty acids as low as possible and cholesterol $<200 \mathrm{ml}$ per day. Fibre intake should be $14 \mathrm{~g} / 1000 \mathrm{kcal}$, with emphasis on low Gl foods. ${ }^{1,3}$

The emphasis that was placed on increasing fat, especially MUFA, stemmed from research associating hypertriglyceridaemia with diets high in carbohydrate. ${ }^{7}$ By decreasing the carbohydrate contribution and simultaneously increasing the fat contribution, especially MUFA, a positive outcome on lipid management, together with glucose control was found and this contributed to the changed recommendations. It was then thought that these high fat diets could result in weight gain, but studies proved that increased MUFA diets as part of an energy-controlled diet plan, would not result in weight gain. ${ }^{2}$ The most recent guidelines take emphasis away from specific percentage contributions of carbohydrate and fat, and make room for a more individualised approach, but they do indicate minimum carbohydrate and maximum saturated fat intake. Also, current 
Table I: Adaptations in the nutritional recommendations of the American Diabetes Association 1994-2006

\begin{tabular}{|l|l|l|l|l|}
\hline Year & Carbohydrate & Fibre & Glycaemic index & Fat \\
\hline $1994^{5}$ & $50-60 \%$ TE & Consume a variety of foods & No recommendation & $\begin{array}{l}\text { TF }<30 \% \text { TE } \\
\text { SF }<10 \% \text { TE }\end{array}$ \\
\hline $2004^{6}$ & CHO + MUFA $=60-70 \%$ TE & Similar to general public & No recommendation & $\begin{array}{l}\text { SF }<7 \% \text { TE } \\
\text { TFA minimum } \\
\text { PUFA }<10 \% \text { TE } \\
\text { Chol }<300 \mathrm{mg}\end{array}$ \\
\hline $2006^{1}$ & CH0 $>130 \mathrm{~g}$ per day & 14 g/1000 kcal & Emphasis on low Gl foods & $\begin{array}{l}\text { SF }<7 \% \text { TE } \\
\text { TFA minimum } \\
\text { Chol }<200 \text { mg }\end{array}$ \\
\hline
\end{tabular}

$\mathrm{TE}=$ total energy; $\mathrm{CHO}=$ carbohydrate; $\mathrm{Gl}=$ glycaemic index $; \mathrm{TF}=$ total fat; $\mathrm{SF}=$ saturated fat; MUFA = monounsaturated fat; $\mathrm{PUFA}=$ poly-unsaturated fat; Chol = cholesterol; $\mathrm{TFA}=$ trans fatty acids

recommendations define better the amount of fibre and place emphasis specifically on Gl.

It is therefore to be noted that the composition of enteral formulae specific for the management of DM reflect the prevailing recommendations at the time a given formula became available. The majority of enteral nutrition products for DM have a carbohydrate content of $30-45 \%$ and fat between $40-49 \%$, mainly monounsaturated fat, with a mix of soluble and insoluble fibre (total of 14-24 g/l). Does this composition of such products have shortand long-term benefits for the patient and which component is crucial for the outcome or is it the combination that really matters?

\section{The role of fibre}

Fibre is advantageous to the diabetic patient not only because it helps against constipation (insoluble fibre), ${ }^{8,9}$ but also because it is known to decrease blood glucose and lipid levels (mainly soluble fibre). ${ }^{8,9}$ This is achieved by the ability of fibre to regulate the absorption of glucose through reducing the rate of digestion and prolonging gastric emptying, thereby improving postprandrial blood glucose control. ${ }^{10}$ The recommended fibre intake varies from 20-50 grams per day, or $14-25 \mathrm{~g} / 1000 \mathrm{kcal}(4200 \mathrm{~kJ})$ and about $50 \%$ of fibre should be of the soluble type. ${ }^{1,7,10} \mathrm{An}$ adequate fluid intake is essential in patients receiving fibre-containing enteral nutrition, especially if long-term, to prevent constipation. ${ }^{11}$

Fibre supplementation of enteral nutrition is normally done in order to attempt to improve GIT tolerance i.e. improve diarrhoea; prevent constipation and improve glycaemic control. ${ }^{8,12}$

Elia et $\mathrm{al}^{13}$ performed a systematic review and meta-analysis to determine the clinical significance of fibre-containing enteral formulae. They included 51 studies that met their inclusion criteria. The type of fibre used most in the studies was soy polysaccharides, followed by a fibre-mix of soluble and insoluble fibres or by other individual fibre components. In the hospitalised patients, fibrecontaining feeds were well tolerated and resulted in improvements in clinical outcomes. The overall incidence of diarrhoea was significantly reduced by $32 \%(O R=0,68, p=0,03)$. The effect was most pronounced in those with a high incidence of diarrhoea at initiation of the formula. Due to heterogeneity between the studies, especially between the ICU studies, results should be interpreted according to ICU and non-ICU patient populations. In the ICU group, the incidence of diarrhoea was reduced by only $2 \%(O R=0,98)$, but in the non-ICU group it was reduced by $58 \%(O R=0,42, p=0,001)$. This highlights the importance of group-specific analysis of data.

The amount of fibre consumed in the studies ranged from 14-34,9 g/day and did not show a significant relationship with the diarrhoea incidence. ${ }^{13}$ The fibre intake did, however, show a significant positive relationship with faecal mass. The latter did not directly affect the prevalence of constipation, since even though the fibre-containing feeds resulted in less patients reporting constipation, the results were not significant. Bowel frequency was increased by fibre supplementation in patients with a low frequency at baseline and vice versa, with little effect on patients with a normal bowel frequency. ${ }^{13}$

When assessing outcomes of fibre-supplemented formulae versus an enhanced fibre intake in the diet, it should be remembered that modified fibres are being used in formulae in order to reduce the occurrence of blockage of feeding tubes. This might affect the metabolic pathways and water-holding properties of the fibre that could explain the differences in outcomes. ${ }^{13}$ Also, assessing formulae using a single fibre source with those using fibre mixtures will also be expected to affect the outcome.

\section{Carbohydrate and fat contribution to total energy}

Some clinical studies have indicated that high carbohydrate (> 55\%), low fat $(<30 \%)$ diets resulted in increased postprandrial plasma glucose and hypertriglyceridaemia as compared to lower carbohydrate (40-45\%) and higher fat (45\%), specifically monounsaturated fat (20-25\%) diets. ${ }^{1,7}$ The latter diet composition did not only result in better glucose control, but the lipid abnormalities were also improved. Other researchers found similar results, but additionally reported the total energy intake of the diet to be the deciding factor. In patients receiving reduced energy intake, high carbohydrate diets did not increase triglyceride levels. This was only found when a high energy intake was maintained. Because of the individual response to high carbohydrate diets, it is recommended that individual dietary adaptations should be made regarding the carbohydrate and fat composition in the diet of a given patient. Other researchers have reported that replacing saturated fats with polyunsaturated fat had similar results to replacing saturated fat with monounsaturated fats. Depending on the total energy and carbohydrate intake, as well as the individual response to the diet, saturated fat can therefore be replaced by either unsaturated fatty acid or carbohydrate. ${ }^{1,2,7}$ 
Studies comparing diabetes-specific formulae with standard formulae have reported a neutral effect on glucose control and lipid management ${ }^{14}$; a reduction in peak glucose values ${ }^{15,16,17}$; a reduced HbA1C profile ${ }^{15,16}$; lower postprandial glucose values ${ }^{18}$; reduced insulin requirements ${ }^{16}$ and no significant effect on lipid values..$^{14,16,18}$

Unfortunately, due to relatively small patient numbers and short duration of studies, as well as different feed compositions, it is difficult to compare results and to determine the clinical impact of the outcomes.

Elia et $\mathrm{al}^{19}$ performed a systematic review and meta-analysis to determine the benefits of enteral nutrition support, specifically the use of diabetes-specific formulae for patients with DM. In total 23 studies were included of which 19 were RCT and the majority studied type $2 \mathrm{DM}$. Due to different study methodologies, relatively small numbers of studies were grouped together for subgroup analysis. Compared to standard formulae, DM specific formulae Studies comparing diabetes-specific formula with standard formula resulted in significantly lower postprandrial blood glucose rise (by $1,03 \mathrm{mmol} / \mathrm{L}$ ), significantly smaller area under the curve (AUC) and insulin AUC values, and reductions in A1c by $0,6 \%$. The effects on the blood lipid profile were less pronounced with no significant effects on cholesterol, LDL, HDL and triglyceride values. Also of importance was the finding that no significant differences in complication rates were reported. The authors concluded that DM specific formulae can result in improvements on blood glucose control. ${ }^{19}$

\section{Conclusion}

Do we really need specialised formulae for diabetes patients or can the patients be treated just as effectively with a standard polymeric formula with or without fibre? According to the $A D A,{ }^{3}$ various studies have attempted to identify the ideal mix of macronutrients for patients with DM. It is unlikely that one such combination of macronutrients exists and therefore emphasis should be placed in individualised approaches. ${ }^{1,3}$

The majority of diabetes-specific enteral formulae on the market in South Africa contain a combination of different fibres, with an altered carbohydrate to fat ratio with emphasis on MUFA. The question can thus be asked which of these two main alterations i.e. addition of fibre or carbohydrate to fat manipulation is the most important and successful, or is it the combination of the two that is of the essence?

In all patients, fermentable fibres are effective for glucose control. 8,9 Since the dyslipidaemia of diabetes (type 2), especially hypertriglyceridaemia, is often disproportionate to the degree of hyperglycaemia, the latter needs to be monitored and treated individually. ${ }^{2}$ Both manipulations of DM-specific enteral formula, i.e. addition of fibre and altered carbohydrate to fat percentage does not show convincing evidence regarding lipid management.,19

In the critically ill patient in an ICU setting, the tolerance of fibre sources is dependent on the function of the gastro-intestinal tract and the use of fibre (quantity and type) should be reconsidered based on the given clinical setting. Patients at high risk of bowel dysmotility and hypotensive patients at risk of developing bowel ischaemia should not receive any fibre, especially insoluble fibre. ${ }^{4,16}$ Since bowel dysmotility is present in a great number of ICU patients, the use of most fibre-containing feeds would not be indicated.

In the non-ICU patient population in a general ward or in the long-term home enterally supported patient the scenario can be totally different. Diarrhoea and/or constipation are probably the most commonly encountered complications in these cases. Fibre containing feeds have been shown to be effective in the management of both extremes of stool adaptations and therefore have a definite role to play. ${ }^{8}$ To prevent and manage diarrhoea, soluble fibre should be administered and to prevent and manage constipation, a mixture of soluble and insoluble fibre is recommended. ${ }^{8,9}$ In the DM patient with gastroparesis, fibre blends, especially insoluble fibre, is not very practical ${ }^{11}$ and a high fat intake which normally may have beneficial outcomes on glucose management due to slowing down of gastric emptying, will also worsen the gastroparesis. ${ }^{20}$

\section{References}

1. American Diabetes Association Position Statement. Nutrition Recommendations and Interventions for Diabetes. Diab Care 2008;31(suppl 1):S61-S78.

2. Wright J. Total parenteral and enteral nutrition in diabetes. Curr Opin Nutr Metab Care 2000;3:5-10.

3. American Diabetes Association. Standards of Medical Care in Diabetes - 2009. Diab Care 2009;32 (suppl 1):S13-S61.

4. Martindale RG, et al. Guidelines for the provision and assessment of nutrition support therapy in the adult critically ill patient: Society of Critical Care Medicine and the American Society for Parenteral and Enteral Nutrition. Crit Care Med 2009;37:1-30

5. American Diabetes Association. Nutrition recommendations and principles for people with Diabetes Mellitus. Diab Care 1994;17:519-522

6. American Diabetes Association. Nutrition Principles and Recommendations in Diabetes. Diab Care 2004;27(suppl 1):S36-S46.

7. Franz MJ. Nutritional management of diabetes mellitus and the dysmetabolic syndrome. Clin Nutr Highlights 2006;2:2-7

8. Meier R \& Gassull MA. Consensus recommendations on the effects and benefits of fibre in clinical practice. Clin Nutr Supplements 2004;1:73-80

9. Alvarez EE \& Sanchez PG. Dietary fibre. Nutr Hosp 2006;21(suppl2):60-71.

10. Canadian Diabetes Association 2008 Clinical Practice Guidelines for the Prevention and Management of Diabetes in Canada. Can J Diab 2008;32(suppl 1):S1-S201

11. Chen $Y$ \& Peterson SJ. Enteral Nutrition Formulas:Which formula is right for your adult patient? Nutr Clin Pract 2009;24:344-355.

12. Cabre E. Fibre supplementation of enteral formula-diets: a look to the evidence. Clin Nutr Supplements 2004;1:63-71

13. Elia M, et al. Systematic review and meta-analysis: the clinical and physiological effects of fibrecontaining enteral formulae. Aliment Pharmacol Ther 2008;27:120-145.

14. Leon-Sonz M, et al. Glycemic and lipid control in hospital type 2 Diabetic patients. Evaluation of 2 enteral nutrition formulas (Low carbohydrate-High monounsaturated fat vs high carbohydrate). JPEN $2005 ; 29: 21-29$

15. Hofman Z, et al. Diabetes specific tube feed results in improved glycaemic and triglyceridaemic control during $6 \mathrm{~h}$ continuous feeding in diabetes patients. Eur J Clin Nutr Metab 2007;2:44-50.

16. Pohl M, et al:. Glycaemic control in type II diabetic tube-fed patients with a new enteral formula low in carbohydrates and high in monounsaturated fatty acids: a randomised controlled trial. Eur J Clin Nutr 2005:59:1221-1232.

17. Ceriello A, et al. Administration of a new diabetes-specific enteral formula results in an improved $24 \mathrm{~h}$ glucose profile in type 2 diabetic patients. Diab Research Clin Pract 2009;84:259-266.

18. Crespillo MDC, et al. Metabolic effects of an enteral nutrition formula for diabetes: comparison with standard formulas in patients with type 1 diabetes. Clin Nutr 2003;22:483-487.

19. Elia M, et al. Enteral Nutritional Support and Use of Diabetes-Specific Formulas for Patients with Diabetes. Diab Care 2005;28:2267-2279.

20. Shakil A, et al. Gastrointestinal complications of Diabetes. Am Fam Physician 2008;77:1697-1702. 